\title{
Developing Sample Management Tools for the Irradiation Test Area (ITA)
}

\author{
Morgan Logsdon ${ }^{1}$
}

\author{
${ }^{1}$ Undergraduate SIST Intern, College of William \& Mary
}

\begin{abstract}
The ITA Data Manager (IDM) has been ported from CERN's Proton Irradiation Facility (IRRAD), where it was used previously as the IRRAD Data Manager (IDM), in order to meet the data management and sample tracking needs of the Irradiation Test Area (ITA), a newly developed facility at Fermilab (launching Fall 2020) operating under the Fermilab Test Beam Facility.
\end{abstract}

\section{INTRODUCTION}

The Fermilab Test Beam Facility (FTBF) and Accelerator Division have been building a new Irradiation Test Area (ITA) that provides Fermilab scientists and engineers with the ability to test sample performance in a comparable environment to Fermilab's LINAC proton beam. Beam intensities increase over time as high-energy physics becomes increasingly high-energy, and continue to produce growing amounts of radiation inside beam environments. Electronic devices placed inside the beam environment for experiment purposes must be able to withstand these high levels of radiation. The ITA development project grew out of a need to provide users with the ability to test device samples under high-intensity radiation and correct for radiation levels comparable to those present in the beam environment.

Physicists and engineers can compensate for the effects of radiation in beam environments by ensuring their electronic devices, sensors, and other experiment components are sufficiently radiation hard. Users and experimentalists must be able to test device performance under the same irradiation as the samples would experience under the beam. Fermilab's high-energy beam users require greater availability and higher intensities in irradiation testing than those offered by existing radiation test facilities. Los Alamos National Laboratory (LANL) and CERN are two notable laboratories with such testing sites, but neither is easily accessible to Fermi users. At LANL, in addition to finding a way to ship samples across the country, users are limited to only two available testing slots per year. CERN's facility, no more accessibly located than LANL's, is currently shut down.

The ITA is designed to provide onsite, highly available irradiation testing to Fermi users. By redirecting part of Fermilab's LINAC beam, ITA utilizes a $400 \mathrm{MeV}$ proton beam and provides fluences of up to $10^{16}$ protons $/ \mathrm{cm}^{2}$ in only four hours. Available 40 times per year, the ITA combines high intensity with high availability that other existing facilities cannot provide.

With the ITA still under construction, the FTBF team has been preparing for its launch in the Fall of 2020. As of Summer 2020, the ITA regulates testing with a new-to-Fermilab data management tool, ITA Data Manager (IDM).

\section{PROJECT GOALS}

The original IDM comes from CERN's Proton Irradiation Facility (IRRAD). Though currently shut down, IRRAD is functionally similar to the ITA and was used to test devices under beams comparable to those at CERN. At IRRAD, the IRRAD Data Manager (IDM), a web-based data management tool, administered the facility's data and streamlines the testing process. The IDM collects and stores data about user credentials, specific experiment needs, dosimeter identification and dosimetry results, user availability, and testing progress. It also allows users to assign their electronic samples to an experiment and logs sample sizes and compounds for the IDM to calculate radiation lengths and cooldown times. 
Over the course of Summer 2020, the IDM has been imported and adapted for use at the ITA in collaboration with CERN and the FTBF group.

\section{PROCESS}

My original goals were to help the FTBF team understand the IDM by identifying helpful functions, and to outline necessary changes for future developers. In order to track progress through achievable milestones, I organized my project into three phases and ensured each phase had at least one deliverable to complete. Initially, I needed to thoroughly understand both the functions of the IDM and the process of the ITA so that I could adapt the IDM to best support ITA needs. I analyzed these IDM functions and identified which componenets are relevant to the ITA, and whether there were nonexistent features that needed to be developed and added on. Next, I focused on deveopling (or editing out) some of these features in the Python code, and documenting any additionally-needed changes for future IDM development.

In order to meet all of these goals, I divided my project into three phases of IDM development and made sure each one had a relevant deliverable. Among three phases of development, I catalogued the files we received from CERN, documented IDM's functions along with required changes, and updated the IDM's stopping power calculations with Mathematica.

\section{Phase I}

The IDM is built in Django, a framework that simplifies web development. Based in Python, Django's built-in features comprise the foundations of many of the IDM's forms and capabilities (1). In the first developmental phase I built an introductory Django knowledge and applied that in order to make changes to titles, toolbars, linked sites, and other small changes that were necessary to begin the shift from the IRRAD Data Manager and the ITA Data Manager.

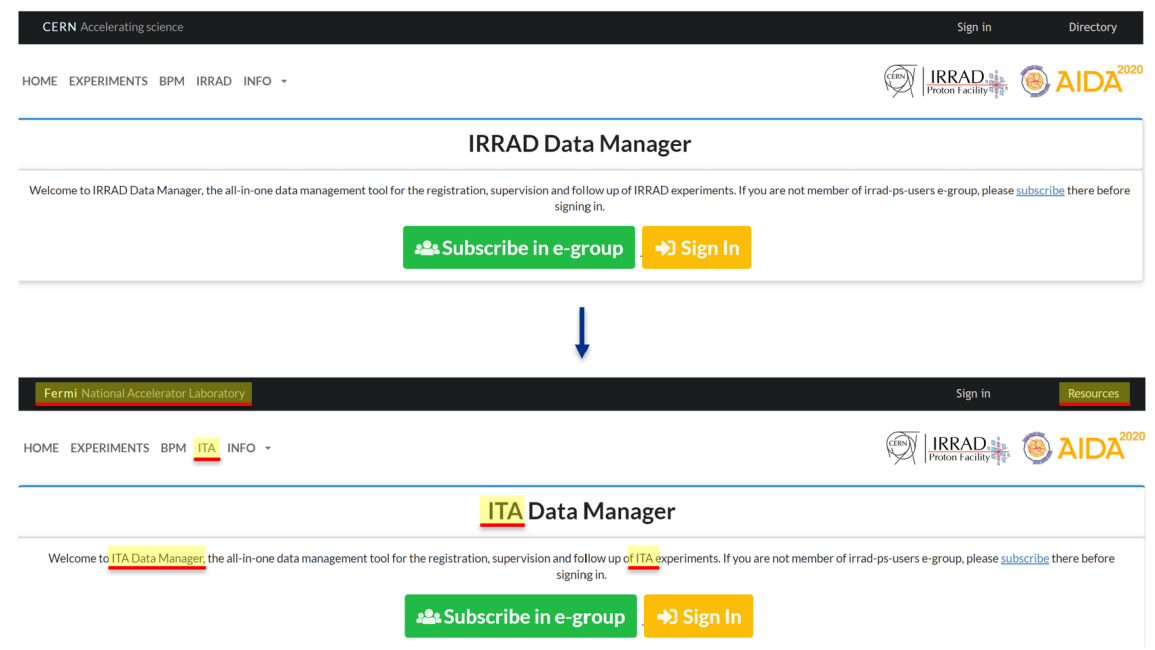

Figure 1. Progression shows initial changes made to the IDM toolbars, titles, etc.

The IDM code received from CERN contained Python and HTML files that were minimally commented and fairly raw, so I initially I spent much of my time learning Django's basic framework and exploring the code files. I also focused my efforts on adding comments, particularly to the Python code, to clarify the files' functions. In order to organize these files, I catalogued them and added brief descriptions of each file's general purpose, noting important functions or classes. 


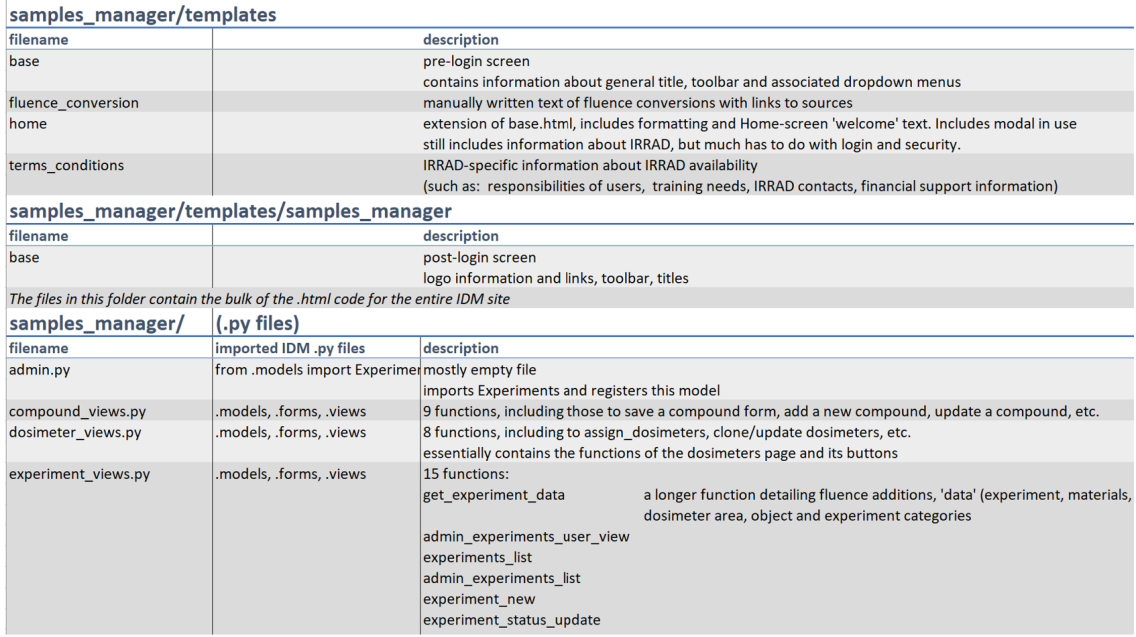

Figure 2. Partial screenshot from Code Catalogue, Microsoft Excel.

This organization proved useful when I needed to find and change a particular set of code, because it allowed me to quickly find where similar functions were housed within the many files in the IDM. It also allowed me to identify which original code files were IRRAD-specific and needed significant changes.

\section{Phase II}

After I gained a better understanding of the IDM in the first phase, I started making content changes to shift its use from IRRAD to ITA in the second phase. I began documenting the functions and capabilities of the IDM in each table and form, highlighting changes that needed to be made in each. I then began editing the Python code in IDM's Django-created forms and adding clarifying comments into the code as applicable.

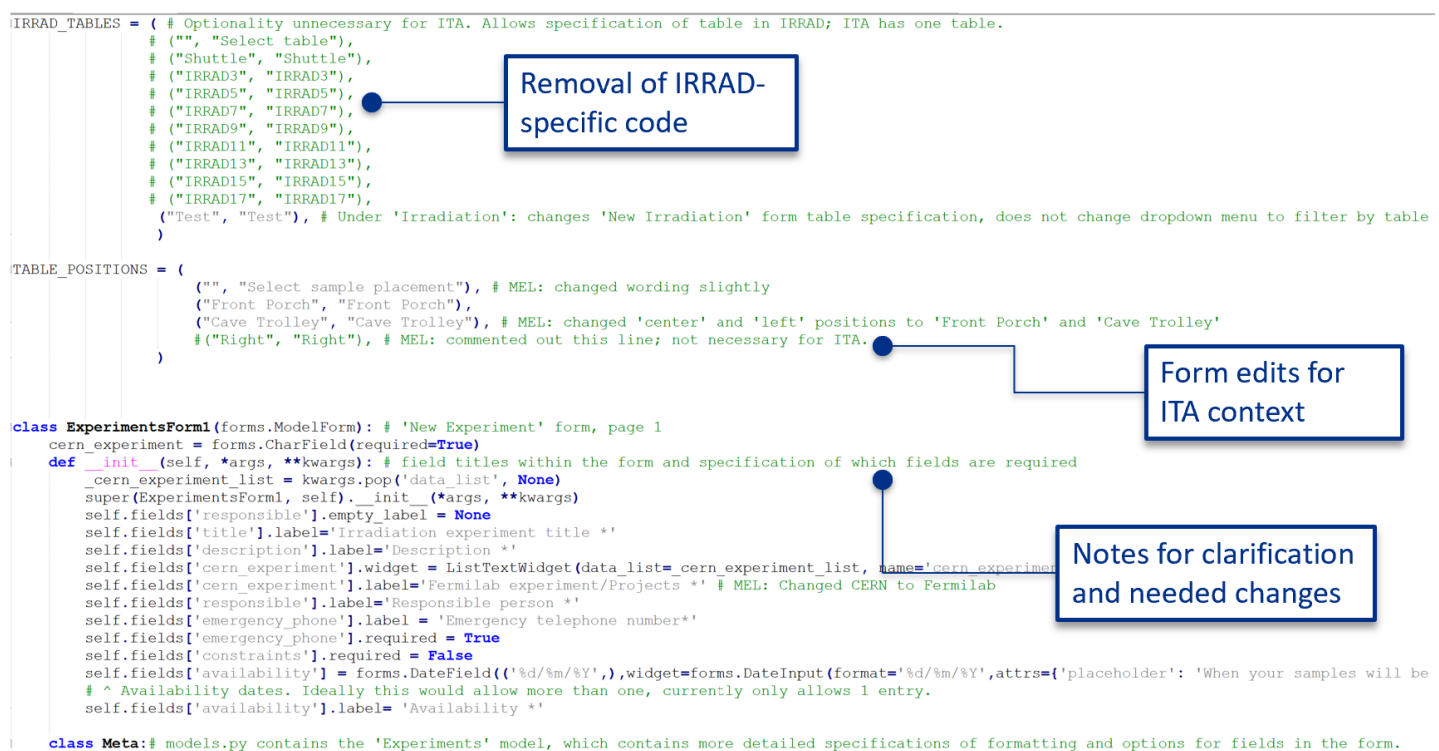

Figure 3. Partial code shows added comments (green), and highlights the three main objectives in working through the IDM code. 
Figure 3 shows the three main purposes of my comments and edits in the code. I frequently removed IRRAD-specific form options or table entries (top box), replaced IRRAD-specific code with an ITArelevant equivalent (center box), or added comments for future developers either to clarify the purpose of the code or to indicate changes that needed to be made.

As I added comments on the Python files, I simultaneously learned about the Linux OS. Throughout this stage, I kept a running log of the changes I made and outlined the pending changes. I also provided a detailed description of the capabilities and functions of the IDM and documented the changes I had personally made to the site. My IDM documentation outlines necessary changes for future IDM development and contains detailed explanations of what I learned about the IDM.

\section{Phase III}

The biggest change that needed to be made in the database behind the IDM was in the stopping power calculations. Stopping power measures energy as transferred from an energetic particle to a medium. For the ITA, stopping power quantifies how much energy is absorbed by a user's sample from the ITA proton beam.

The original database table containing data for materials and elements had stopping power calculations done for 97 elements, but the values had been calculated for the energy and particles of CERN's IRRAD beams. With the help of Dr. Michael Geelhoed, who provided me with both guidance and resources such as James E. Turner's textbook Atoms, Radiation, and Radiation Protection and Fermilab Report 1834 (Radiation Physics for Personnel and Environmental Protection), I learned how to calculate stopping power. Then I created a Mathematica notebook to compute these values for all 97 of the original elements and specifically for the ITA's $400 \mathrm{MeV}$ proton beam. In order to complete these calculations, I used the equation

$$
-\frac{d E}{d x}=0.3071 z^{2} \frac{Z}{A} \frac{1}{\beta} \log \frac{1.022 \times 10^{6} \gamma^{2} \beta}{I}-\beta-\log \gamma
$$

where $Z, A, I$ represent the atomic number, atomic mass, and excitation energy of each element, individually. $z, \beta, \gamma$ represent the atomic number, the ratio of the particle velocity to the speed of light, and relativistic constant $\frac{1}{\sqrt{1-\beta^{2}}}$, respectively, of the beam particles (2).

As I iterated through the 97 elements in the table and updated it according to my calculations, I referred to the Stopping Power and Range Tables for Protons (pstar) resource provided by the National Institute of Standards and Technology (NIST). Though pstar does not include all 97 of the elements, I was able to audit my calculations by comparing trends and values to ensure my calculations are accurate.

\begin{tabular}{|c|c|c|c|}
\hline \multirow{2}{*}{$\begin{array}{c}\text { (required) } \\
\text { Kinetic Energy } \\
(\mathrm{MeV})\end{array}$} & \multicolumn{3}{|c|}{ Stopping Power $\left(\mathrm{MeV} \mathrm{cm}^{2} / \mathrm{g}\right)$} \\
\hline & Electronic & Nuclear & Total \\
\hline $1.000 \mathrm{E}-03$ & $7.465 \mathrm{E}+02$ & $2.264 \mathrm{E}+02$ & $9.730 \mathrm{E}+02$ \\
\hline $1.500 \mathrm{E}-03$ & $9.143 \mathrm{E}+02$ & $1.726 \mathrm{E}+02$ & $1.087 \mathrm{E}+03$ \\
\hline $2.000 \mathrm{E}-03$ & $1.056 \mathrm{E}+03$ & $1.411 \mathrm{E}+02$ & $1.197 \mathrm{E}+03$ \\
\hline $2.500 \mathrm{E}-03$ & $1.180 \mathrm{E}+03$ & $1.201 \mathrm{E}+02$ & $1.300 \mathrm{E}+03$ \\
\hline $3.000 \mathrm{E}-03$ & $1.293 \mathrm{E}+03$ & $1.050 \mathrm{E}+02$ & $1.398 \mathrm{E}+03$ \\
\hline $4.000 \mathrm{E}-03$ & $1.493 \mathrm{E}+03$ & $8.454 \mathrm{E}+01$ & $1.578 \mathrm{E}+03$ \\
\hline $5.000 \mathrm{E}-03$ & $1.669 \mathrm{E}+03$ & $7.122 \mathrm{E}+01$ & $1.741 \mathrm{E}+03$ \\
\hline $6.000 \mathrm{E}-03$ & $1.829 \mathrm{E}+03$ & $6.179 \mathrm{E}+01$ & $1.890 \mathrm{E}+03$ \\
\hline $7.000 \mathrm{E}-03$ & $1.975 \mathrm{E}+03$ & $5.472 \mathrm{E}+01$ & $2.030 \mathrm{E}+03$ \\
\hline $8.000 \mathrm{E}-03$ & $2.112 \mathrm{E}+03$ & $4.921 \mathrm{E}+01$ & $2.161 \mathrm{E}+03$ \\
\hline $9.000 \mathrm{E}-03$ & $2.240 \mathrm{E}+03$ & $4.479 \mathrm{E}+01$ & $2.284 \mathrm{E}+03$ \\
\hline $1.000 \mathrm{E}-02$ & $2.361 \mathrm{E}+03$ & $4.115 \mathrm{E}+01$ & $2.402 \mathrm{E}+03$ \\
\hline $1.250 \mathrm{E}-02$ & $2.586 \mathrm{E}+03$ & $3.434 \mathrm{E}+01$ & $2.621 \mathrm{E}+03$ \\
\hline $1.500 \mathrm{E}-02$ & $2.777 \mathrm{E}+03$ & $2.958 \mathrm{E}+01$ & $2.807 \mathrm{E}+03$ \\
\hline $1.750 \mathrm{E}-02$ & $2.942 \mathrm{E}+03$ & $2.605 \mathrm{E}+01$ & $2.968 \mathrm{E}+03$ \\
\hline $2.000 \mathrm{E}-02$ & $3.084 \mathrm{E}+03$ & $2.332 \mathrm{E}+01$ & $3.107 \mathrm{E}+03$ \\
\hline $2.250 \mathrm{E}-02$ & $3.208 \mathrm{E}+03$ & $2.114 \mathrm{E}+01$ & $3.229 \mathrm{E}+03$ \\
\hline $2.500 \mathrm{E}-02$ & $3.316 \mathrm{E}+03$ & $1.936 \mathrm{E}+01$ & $3.335 \mathrm{E}+03$ \\
\hline $2.750 \mathrm{E}-02$ & $3.409 \mathrm{E}+03$ & $1.787 \mathrm{E}+01$ & $3.427 \mathrm{E}+03$ \\
\hline $3.000 \mathrm{E}-02$ & $3.490 \mathrm{E}+03$ & $1.661 \mathrm{E}+01$ & $3.506 \mathrm{E}+03$ \\
\hline
\end{tabular}

Figure 4. Partial chart from NIST's Stopping Power and Range Tables for Protons (pstar). 
In its initial stages the ITA will test CMS and ATLAS samples, which are primarily silicon chips, but having all 97 elements updated allows users to submit samples of a variety of chemical properties and compositions. Now, in preparation for those test, the IDM has stored the updated table, ready for use.

\section{CONCLUSION}

In anticipation of the ITA's launch in Fall 2020, my summer work has been handed over to the FTBF group. The IT team has worked behind the scenes to incorporate Fermilab's authentication and security systems, and preparing the IDM for deployment. The FTBF group will be finishing the changes outlined in my documentation and preparing to launch the IDM alongside the ITA.

\section{ACKNOWLEDGMENTS}

I'd like to thank my supervisor, Dr. Niner, for answering my questions with patience and for allowing me the independence to manage my project while offering all the support that I needed to do so.

Dr. Geelhoed has been tremendously helpful in my project, both in our weekly group mentoring sessions and by providing me with resources and advice, especially toward the end of my project.

This manuscript has been authored by Fermi Research Alliance, LLC under Contract No. DE-AC0207CH11359 with the U.S. Department of Energy, Office of Science, Office of High Energy Physics.

\section{REFERENCES}

[1] Django Overview. (n.d.). Retrieved July 21, 2020, from https://www.djangoproject.com/start/overview/

[2] Cossairt, J. D. (2015). Radiation Physics for Personnel and Environmental Protection (Revision 14, Fermilab Report TM-1834). Chicago, IL: Fermi National Accelerator Laboratory.

[3] Turner, J. E. (2010). Atoms, Radiation, and Radiation protection (3rd ed.). Weinheim: Wiley-VCH Verlag GmbH amp; KGaA. 